\title{
ON THE APPROXIMATION OF AN INTEGRAL BY A SUM OF RANDOM VARIABLES
}

\author{
JOHN H.J. EINMAHL ${ }^{1}$ \\ Eindhoven University of Technology \\ Department of Mathematics and Computing Science \\ P.O. Box 513, $5600 \mathrm{MB}$ Eindhoven, The Netherlands \\ MARTIEN C.A. VAN ZUIJLEN \\ University of Nijmegen \\ Department of Mathematics \\ Toernooiveld 1, 6525 ED Nijmegen, The Netherlands
}

(Received October, 1996; Revised September, 1997)

\begin{abstract}
We approximate the integral of a smooth function on $[0,1]$, where values are only known at $n$ random points (i.e., a random sample from the uniform- $(0,1)$ distribution), and at 0 and 1 . Our approximations are based on the trapezoidal rule and Simpson's rule (generalized to the nonequidistant case), respectively. In the first case, we obtain an $n^{2}$-rate of convergence with a degenerate limiting distribution; in the second case, the rate of con-vergence is as fast as $n^{3^{1} / 2}$, whereas the limiting distribution is Gaussian then.
\end{abstract}

Key words: Numerical Integration, Order Statistics, Spacings.

AMS subject classifications: $60 \mathrm{~F} 05,62 \mathrm{G} 30,65 \mathrm{D} 30$.

\section{Introduction and Main Results}

Suppose we (can) only observe the values of a smooth function $f:[0,1] \rightarrow \mathbb{R}$ at the points $U_{0}, U_{1}, \ldots, U_{n}, U_{n+1}$, where $U_{1}, U_{2}, \ldots, U_{n}$ are the order statistics $\left(U_{1} \leq U_{2} \leq\right.$ $\left.\cdots \leq U_{n}\right)$ of $n$ independent uniformly- $(0,1)$ distributed random variables and $U_{0}:=0$, $U_{n+1}:=1$. It is our aim to estimate the integral

$$
I:=\int_{0}^{1} f(x) d x
$$

from these observations, i.e., by only using $\left(U_{i}, f\left(U_{i}\right)\right), i=0,1, \ldots, n+1$. The first

\footnotetext{
${ }^{1}$ Research partially supported by European Union HCM grant ERB CHRX-CT 940693.
}

Printed in the U.S.A. (C)1998 by North Atlantic Science Publishing Company 
estimator we will employ is constructed by using the 'trapezoidal rule' on each subinterval $\left[U_{i-1}, U_{i}\right], i=1, \ldots, n+1$. This rule approximates an integral $\int_{a}^{b} g(x) d x$ simply by $\frac{1}{2}(b-a)(g(a)+g(b))$ and it can easily be shown (see, e.g., Isaacson and Keller [2], p. 304) that

$$
\frac{1}{2}(b-a)(g(a)+g(b))-\int_{a}^{b} g(x) d x=\frac{1}{12}(b-a)^{3} g^{\prime \prime}(\eta)
$$

where $\eta \in(a, b)$. Writing $D_{i}=U_{i}-U_{i-1}, i=1, \ldots, n+1$, for the spacings of the $U_{i}$ 's, our estimator of $I$ becomes

$$
I_{n}:=\sum_{i=1}^{n+1} \frac{1}{2} D_{i}\left(f\left(U_{i-1}\right)+f\left(U_{i}\right)\right) .
$$

Using (2), we will prove the following limiting result for the standardized difference of $I_{n}$ and $I$ :

Theorem 1: If $\left|f^{\prime \prime \prime}\right|$ is bounded, then

$$
n^{2}\left(I_{n}-I\right) \stackrel{P}{\rightarrow} \frac{1}{2}\left(f^{\prime}(1)-f^{\prime}(0)\right), \quad \text { as } n \rightarrow \infty .
$$

A much better and probabilistically more interesting estimator is obtained by applying a 3-points formula, i.e., for a given $c \in(a, b)$, we approximate $\int_{a}^{b} g(x) d x$ by $w_{1} g(a)+w_{2} g(c)+w_{3} g(b)$ in such a way that the approximation error is zero in the case $g$ is a polynomial of second degree. If the 3 points are equidistant, this approximation is known as Simpson's rule. It is not hard to show that

$$
w_{1}=\frac{1}{6}(b-a)\left(2-\frac{b-c}{c-a}\right), w_{2}=\frac{1}{6} \frac{(b-a)^{3}}{(c-a)(b-c)}, w_{3}=\frac{1}{6}(b-a)\left(2-\frac{c-a}{b-c}\right)
$$

and it follows (see again Isaacson and Keller [2], p. 304) that

$w_{1} g(a)+w_{2} g(c)+w_{3} g(b)-\int_{a}^{b} g(x) d x=-\frac{1}{6} \int_{a}^{b}(x-a)(x-c)(x-b) g^{(3)}(\eta) d x$

where $\eta=\eta(x) \in(a, b)$. Hence, our estimator of $I$ in (1), again denoted by $I_{n}$, becomes

$$
\begin{aligned}
& I_{n}=\sum_{i=1}^{\frac{n+1}{2}} \frac{1}{6}\left(D_{2 i-1}+D_{2 i}\right)\left\{\left(2-\frac{D_{2 i}}{D_{2 i-1}}\right) f\left(U_{2 i-2}\right)\right. \\
& \left.+\frac{\left(D_{2 i-1}+D_{2 i}\right)^{2}}{D_{2 i-1} D_{2 i}} f\left(U_{2 i-1}\right)+\left(2-\frac{D_{2 i-1}}{D_{2 i}}\right) f\left(U_{2 i}\right)\right\}
\end{aligned}
$$

where, for convenience, $n$ is taken to be odd. Formula (6) will be used to prove our main result:

Theorem 2: Let $n$ be odd. If $\left|f^{(5)}\right|$ is bounded, then

$$
n^{3 \frac{1}{2}}\left(I_{n}-I\right) \rightarrow \sqrt{\frac{35}{3} \int_{0}^{1}\left(f^{(3)}(x)\right)^{2} d x} Z \text {, as } n \rightarrow \infty
$$


where $Z$ is a standard normal random variable.

Remark 1: The present techniques can be easily adapted to cover the situation where the $U_{i}$ 's are the order statistics of $n$ independent random variables with common distribution function $G$ (on $(0,1)$ ) having a smooth density $g$. The adaptation is based on the quantile transform, transforming a uniform random variable $V$ into a random variable $G^{-1}(V)$ with distribution function $G$. In this case, under regularity conditions on $g$, we obtain that the weak limit in Theorem 1 becomes $\frac{1}{2} \int_{0}^{1}\left(f^{\prime \prime}(x) / g^{2}(x)\right) d x$ instead of $\frac{1}{2} \int_{0}^{1} f^{\prime \prime}(x) d x=\frac{1}{2}\left(f^{\prime}(1)-f^{\prime}(0)\right)$. In Theorem 2 , the limiting random variable is again centered normal but now the standard deviation becomes

$$
\sqrt{\frac{35}{3} \int_{0}^{1} \frac{\left(f^{(3)}(x)\right)^{2}}{g^{7}(x)} d x}
$$

On the other hand, the uniform distribution seems very relevant because of the following. Since $\int_{0}^{1} f(x) d x$ can be considered as the mean 'output', given that the $x$ values are 'equally important', it seems desirable to estimate $\int_{0}^{1} f(x) g(x) d x=$ $\int_{0}^{1} f\left(G^{-1}(y)\right) d y$ in the case the random variables are distributed according to $G$. But if $G$ is known, we can replace the pairs $\left(U_{i}, f\left(U_{i}\right)\right)$ (just below (1)), with $U_{i}$ 's being the order statistics from $G$, by $\left(G\left(U_{i}\right), f\left(U_{i}\right)\right)=\left(G\left(U_{i}\right), f\left(G^{-1}\left(G\left(U_{i}\right)\right)\right)\right)$. This brings us back to the 'uniform distribution setup' with $f$ replaced by $f \circ G^{-1}$, but that is just the function whose integral we wanted to estimate as argued above!

This idea leads to possible ways of applying the results. Suppose $U_{i}$ represents some uncontrollable physical random quantity, like temperature, humidity or light intensity with a known distribution function $G$ having density $g$. Suppose also that we can measure $f$ (the output or yield) only at the $U_{i}$ and that we are interested in the mean output $I_{g}=\int_{0}^{1} f(x) g(x) d x$. Then one can use our theorems to obtain rapidly converging estimators of $I_{g}$. In particular, when measuring the $f$-values is hard or expensive, one can get good estimators based on a few observations.

Also note that for the trapezoidal rule in Theorem 1 and $f^{\prime \prime}$ being constant, the uniform distribution is optimal, since $\int_{0}^{1} g^{-2}(x) d x \geq \int_{0}^{1} 1 d x=1$. (This can be easily
seen by using Jensen's inequality:

$$
\begin{aligned}
\int_{0}^{1} \frac{1}{g^{2}(x)} d x & =\int_{0}^{1} \frac{1}{g^{3}(x)} g(x) d x=\mathbb{E} \frac{1}{g^{3}(X)} \geq\left(\mathbb{E} \frac{1}{g(X)}\right)^{3} \\
& =\left(\int_{0}^{1} \frac{1}{g(x)} g(x) d x\right)^{3}=1
\end{aligned}
$$

where $X$ is a random variable with density g.) A similar remark applies to Theorem 2 with $f^{(3)}$ being constant.

Remark 2: There are various other ways to extend our results, which we will not pursue here, e.g., applying $m$-points formulas for $m>3$ (Simpson's rule is 'by far the 
most frequently used in obtaining approximate integrals', Davis and Rabinowitz [1], p. 45), combining trapezoidal rules to eliminate the bias $\frac{1}{2}\left(f^{\prime}(1)-f^{\prime}(0)\right)$, proving a 'second order' limit result for $n^{2}\left(I_{n}-I\right)-\frac{1}{2}\left(f^{\prime}(1)-f^{\prime}(0)\right)$ in Theorem 1 , or treating the case $n$ 'even' in Theorem 2. We are not pursuing these extensions because we believe they are not very interesting and/or they do not give good results.

Remark 3: We briefly compare our results with the deterministic, equidistant case, i.e., $U_{i}=\frac{i}{n+1}, i=0,1, \ldots, n+1$. It is well-known that the limit in Theorem 1 is $\frac{1}{12}\left(f^{\prime}(1)-f^{\prime}(0)\right)$ in that case, which means that we loose a factor of 6 by having random $U_{i}$ 's. (Essentially, this 6 is coming from the third moment of a standard exponential random variable.) From Theorem 2, it is well-known that in the equidistant case (Simpson's rule), the rate is $n^{4}$. So, there our loss is of order $n^{1 / 2}$. Nevertheless, from statistical point of view, $n^{3 \frac{1}{2}}$ is a remarkably fast rate of convergence.

\section{Proofs}

The following well-known lemma will be used frequently; it can be found in, e.g., Shorack and Wellner [3], p. 721.

Lemma 1: Let $E_{1}, \ldots, E_{n+1}$ be independent exponential random variables with mean 1 and $S_{n+1}$ be their sum. With $D_{i}, i=1, \ldots, n+1$, as before, we have

$$
\left(D_{1}, \ldots, D_{n+1}\right) \stackrel{d}{=}\left(\frac{E_{1}}{S_{n+1}}, \ldots, \frac{E_{n+1}}{S_{n+1}}\right) .
$$

Proof of Theorem 1: Using (3), (1) and (2) we see that

for some $\tilde{U}_{i} \in\left(U_{i-1}, U_{i}\right)$, and hence,

$$
n^{2}\left(I_{n}-I\right)=\frac{n^{2}}{12} \sum_{i=1}^{n+1} D_{i}^{3} f^{\prime \prime}\left(\widetilde{U}_{i}\right)
$$

$$
n^{2}\left(I_{n}-I\right)=\frac{n^{2}}{12} \sum_{i=1}^{n+1} D_{i}^{3} f^{\prime \prime}\left(\frac{i}{n+1}\right)+\frac{n^{2}}{12} \sum_{i=1}^{n+1} D_{i}^{3}\left(\widetilde{U}_{i}-\frac{i}{n+1}\right) f^{\prime \prime \prime}\left(\widetilde{\widetilde{U}}_{i}\right)
$$

with $\widetilde{\widetilde{U}}_{i}$ between $\widetilde{U}_{i}$ and $\frac{i}{n+1}$. From the boundedness of $\left|f^{\prime \prime \prime}\right|$ (by $M$, say) and the weak convergence (to a Brownian bridge) of the uniform quantile process (see, e.g., Shorack and Wellner [3]), it is readily seen that

$$
\begin{aligned}
& \left|\frac{n^{2}}{12} \sum_{i=1}^{n+1} D_{i}^{3}\left(\tilde{U}_{i}-\frac{i}{n+1}\right) f^{\prime \prime \prime}\left(\tilde{\widetilde{U}}_{i}\right)\right| \\
& \leq \frac{n^{2}}{12} M \sup _{i \in\{1, \ldots, n+1\}}\left|\tilde{U}_{i}-\frac{i}{n+1}\right| \sum_{i=1}^{n+1} D_{i}^{3}=O_{p}\left(n^{1 \frac{1}{2}}\right) \sum_{i=1}^{n+1} D_{i}^{3} .
\end{aligned}
$$

But

$$
\sum_{i=1}^{n+1} D_{i}^{3} \stackrel{d}{=} \frac{1}{S_{n+1}^{3}} \sum_{i=1}^{n+1} E_{i}^{3}
$$

by Lemma 1 , and by two applications of the weak law of large numbers, this last expression is $O_{p}\left(n^{-2}\right)$. Combining this with (10) and (11) yields that the second term on the right in (9) converges to zero in probability. Hence, it remains to consider the first term 


$$
\frac{n^{2}}{12} \sum_{i=1}^{n+1} D_{i}^{3} f^{\prime \prime}\left(\frac{i}{n+1}\right) \stackrel{d}{=} \frac{1}{12 n}\left(\frac{n}{S_{n+1}}\right)^{3} \sum_{i=1}^{n+1} f^{\prime \prime}\left(\frac{i}{n+1}\right) E_{i}^{3}
$$

or, since $\left(n / S_{n+1}\right)^{3} \stackrel{P}{\rightarrow} 1$,

$$
\frac{1}{12 n} \sum_{i=1}^{n+1} f^{\prime \prime}\left(\frac{i}{n+1}\right) E_{i}^{3}
$$

By Chebysev's inequality, it follows that

$$
\frac{1}{12 n} \sum_{i=1}^{n+1} f^{\prime \prime}\left(\frac{i}{n+1}\right) E_{i}^{3}-\frac{1}{2 n} \sum_{i=1}^{n+1} f^{\prime \prime}\left(\frac{i}{n+1}\right) \stackrel{P}{\rightarrow} 0 .
$$

The proof is complete by noting that

$$
\frac{1}{2 n} \sum_{i=1}^{n+1} f^{\prime \prime}\left(\frac{i}{n+1}\right) \rightarrow \frac{1}{2} \int_{0}^{1} f^{\prime \prime}(x) d x=\frac{1}{2}\left(f^{\prime}(1)-f^{\prime}(0)\right) .
$$

The proof of Theorem 2 is heavily based on the following two lemmas.

Lemma 2: Let $E_{1}, \ldots, E_{n+1}, \quad n$ odd, be independent exponential random variables with mean 1 . Write

$$
\begin{gathered}
X_{i}=\left(E_{2 i-1}+E_{2 i}\right)^{3}\left(E_{2 i}-E_{2 i-1}\right), \quad i=1,2, \ldots, \frac{n+1}{2}, \\
Y_{i}=X_{i} \sum_{j=1}^{2 i-2}\left(E_{j}-1\right), \quad i=2,3, \ldots, \frac{n+1}{2} .
\end{gathered}
$$

Then,

$$
\mathbb{E} X_{i}=0, \operatorname{Var} X_{i}=120960, \mathbb{E} Y_{i}=0, \operatorname{Var} Y_{i}=120960(2 i-2),
$$

$$
\operatorname{Cov}\left(Y_{i}, Y_{k}\right)=0, \text { for } i \neq k \text {. }
$$

Proof: By symmetry, we see that $\mathbb{E} X_{i}=0$; a straightforward computation yields $\operatorname{Var} X_{i}=\mathbb{E} X_{i}^{2}=120960$. For the $Y_{i}$ 's we have

$$
\begin{gathered}
\mathbb{E} Y_{i}=\mathbb{E} X_{i} \mathbb{E} \sum_{j=1}^{2 i-2}\left(E_{j}-1\right)=0, \\
\operatorname{Var} Y_{i}=\mathbb{E} Y_{i}^{2}=\mathbb{E} X_{i}^{2} \mathbb{E}\left(\sum_{j=1}^{2 i-2}\left(E_{j}-1\right)\right)^{2} \\
=\operatorname{Var} X_{i} \operatorname{Var}\left(\sum_{j=1}^{2 i-2} E_{j}\right)=120960(2 i-2),
\end{gathered}
$$

and for $i<k$,$$
\operatorname{Cov}\left(Y_{i}, Y_{k}\right)=\mathbb{E} Y_{i} Y_{k}
$$$$
=\mathbb{E} X_{k}\left(\sum_{j=1}^{2 k-2}\left(E_{j}-1\right)\right) X_{i}\left(\sum_{j=1}^{2 i-2}\left(E_{j}-1\right)\right)
$$$$
=\mathbb{E} X_{k} \mathbb{E}\left(\sum_{j=1}^{2 k-2}\left(E_{j}-1\right)\right) X_{i}\left(\sum_{j=1}^{2 i-2}\left(E_{j}-1\right)\right)
$$$$
=0 \text {. }
$$ 
Lemma 3: Under the conditions of Theorem 2, we have, as $n \rightarrow \infty$,

$$
\left|n^{3 \frac{1}{2}}\left(I_{n}-I\right)-\frac{n^{3 \frac{1}{2}}}{72} \sum_{i=1}^{\frac{n+1}{2}} f^{(3)}\left(\frac{2 i-2}{n+1}\right)\left(D_{2 i-1}+D_{2 i}\right)^{3}\left(D_{2 i}-D_{2 i-1}\right)\right|=o_{p}(1) \text {. }
$$

Proof: By (7), (1) and (6) we have $n^{3 \frac{1}{2}}\left(I_{n}-I\right)$

$$
=-\frac{n^{3 \frac{1}{2}}}{6} \sum_{i=1}^{\frac{n+1}{2}} \int_{U_{2 i-2}}^{U_{2 i}}\left(x-U_{2 i-2}\right)\left(x-U_{2 i-1}\right)\left(x-U_{2 i}\right) f^{(3)}\left(\tilde{U}_{2 i}\right) d x
$$

for some $\tilde{U}_{2 i}=\widetilde{U}_{2 i}(x) \in\left(U_{2 i-2}, U_{2 i}\right)$ and hence for some $\tilde{\widetilde{U}}_{2 i}=\tilde{\widetilde{U}}_{2 i}(x) \epsilon$ $\left(U_{2 i-2}, \widetilde{U}_{2 i}\right)$, the right-hand side of $(12)$ is equal to

$$
\begin{gathered}
-\frac{n^{3 \frac{1}{2}}}{6} \sum_{i=1}^{\frac{n+1}{2}} \int_{U_{2 i-2}}^{U_{2 i}}\left(x-U_{2 i-2}\right)\left(x-U_{2 i-1}\right)\left(x-U_{2 i}\right) \\
\quad \times\left(f^{(3)}\left(U_{2 i-2}\right)+\left(\tilde{U}_{2 i}-U_{2 i-2}\right) f^{(4)}\left(\tilde{\widetilde{U}}_{2 i}\right)\right) d x \\
=\frac{n^{3 \frac{1}{2}}}{72} \sum_{i=1}^{\frac{n+1}{2}} f^{(3)}\left(U_{2 i-2}\right)\left(D_{2 i-1}+D_{2 i}\right)^{3}\left(D_{2 i}-D_{2 i-1}\right) \\
-\frac{n^{3 \frac{1}{2}}}{6} \sum_{i=1}^{\frac{n+1}{2}} \int_{U_{2 i-2}}^{U_{2 i}}\left(x-U_{2 i-2}\right)\left(x-U_{2 i-1}\right)\left(x-U_{2 i}\right)\left(\tilde{U}_{2 i}-U_{2 i-2}\right) f^{(4)}\left(\tilde{\widetilde{U}}_{2 i}\right) d x
\end{gathered}
$$

Let $M$ be a bound on $\left|f^{(5)}\right|$ and all lower order derivatives of $f$. Then the absolute value of this last term is bounded from above by

$$
\begin{aligned}
& M \frac{n^{3 \frac{1}{2}}}{6} \sum_{i=1}^{\frac{n+1}{2}} \int_{U_{2 i-2}}^{U_{2 i}}\left(x-U_{2 i-2}\right)\left|x-U_{2 i-1}\right|\left(U_{2 i}-x\right)\left(\widetilde{U}_{2 i}-U_{2 i-2}\right) d x \\
& \leq \frac{M}{6} n^{3 \frac{1}{2}} \sum_{i=1}^{\frac{n+1}{2}}\left(D_{2 i-1}+D_{2 i}\right)^{5} \stackrel{d}{=} \frac{M}{6} n^{3 \frac{1}{2}} \frac{1}{S_{n+1}^{5}} \sum_{i=1}^{\frac{n+1}{2}}\left(E_{2 i-1}+E_{2 i}\right)^{5}=o_{p}(1),
\end{aligned}
$$

due to Lemma 1 and two applications of the weak law of large numbers.

So, it suffices to show the convergence to zero in probability of

$$
\begin{aligned}
& \frac{n^{3 \frac{1}{2}}}{72} \sum_{i=1}^{\frac{n+1}{2}}\left(f^{(3)}\left(U_{2 i-2}\right)-f^{(3)}\left(\frac{2 i-2}{n+1}\right)\right)\left(D_{2 i-1}+D_{2 i}\right)^{3}\left(D_{2 i}-D_{2 i-1}\right) \\
= & \frac{n^{3 \frac{1}{2}}}{72} \sum_{i=2}^{\frac{n+1}{2}}\left(U_{2 i-2}-\frac{2 i-2}{n+1}\right) f^{(4)}\left(\frac{2 i-2}{n+1}\right)\left(D_{2 i-1}+D_{2 i}\right)^{3}\left(D_{2 i}-D_{2 i-1}\right)
\end{aligned}
$$




$$
\begin{aligned}
& +\frac{n^{3 \frac{1}{2}}}{144} \sum_{i=2}^{\frac{n+1}{2}}\left(U_{2 i-2}-\frac{2 i-2}{n+1}\right)^{2} f^{(5)}\left(\bar{U}_{2 i-2}\right)\left(D_{2 i-1}+D_{2 i}\right)^{3}\left(D_{2 i}-D_{2 i-1}\right) \\
& =: T_{1, n}+T_{2, n},
\end{aligned}
$$

for some $\bar{U}_{2 i-2}$ between $U_{2 i-2}$ and $\frac{2 i-2}{n+1}$. By the weak convergence of the uniform quantile process,

$$
\begin{gathered}
\left|T_{2, n}\right| \leq \frac{n^{3 \frac{1}{2}}}{144} M \sup _{i \in\left\{2,3, \ldots, \frac{n+1}{2}\right\}}\left(U_{2 i-2}-\frac{2 i-2}{n+1}\right)^{2} \sum_{i=2}^{\frac{n+1}{2}}\left(D_{2 i-1}+D_{2 i}\right)^{4} \\
=O_{p}\left(n^{2 \frac{1}{2}}\right) \sum_{i=2}^{\frac{n+1}{2}}\left(D_{2 i-1}+D_{2 i}\right)^{4} .
\end{gathered}
$$

By Lemma 1 and twice the weak law of large numbers, this last expression is easily seen to be $o_{p}(1)$. Hence, the proof of Lemma 3 is complete if we show $T_{1, n}=o_{p}(1)$.

From Lemma 1 we obtain

$$
\begin{aligned}
& T_{1, n} \stackrel{d}{=} \frac{n^{3 \frac{1}{2}}}{72} \sum_{i=2}^{\frac{n+1}{2}}\left(\frac{\sum_{j=1}^{2 i-2} E_{j}}{S_{n+1}}-\frac{2 i-2}{n+1}\right) f^{(4)}\left(\frac{2 i-2}{n+1}\right)\left(\frac{E_{2 i-1}+E_{2 i}}{S_{n+1}}\right)^{3}\left(\frac{E_{2 i}-E_{2 i-1}}{S_{n+1}}\right) \\
& \quad=\frac{n^{3 \frac{1}{2}}}{72} S_{n+1}^{-5} \sum_{i=2}^{\frac{n+1}{2}}\left(\sum_{j=1}^{2 i-2}\left(E_{j}-1\right)\right) f^{(4)}\left(\frac{2 i-2}{n+1}\right)\left(E_{2 i-1}+E_{2 i}\right)^{3}\left(E_{2 i}-E_{2 i-1}\right) \\
& +\frac{n^{3 \frac{1}{2}}}{72} S_{n+1}^{-5}\left(1-\frac{S_{n+1}}{n+1}\right) \sum_{i=2}^{\frac{n+1}{2}}(2 i-2) f^{(4)}\left(\frac{2 i-2}{n+1}\right)\left(E_{2 i-1}+E_{2 i}\right)^{3}\left(E_{2 i}-E_{2 i-1}\right) \\
& =: T_{3, n}+T_{4, n} .
\end{aligned}
$$

It is immediate from the central limit theorem for $S_{n+1} /(n+1)$ that

$$
T_{4, n}=O_{p}\left(n^{-2}\right) \sum_{i=2}^{\frac{n+1}{2}}(2 i-2) f^{(4)}\left(\frac{2 i-2}{n+1}\right) X_{i},
$$

where the $X_{i}$ 's are as in Lemma 2. Now using that lemma in conjunction with Chebysev's inequality, it readily follows that $T_{4, n}=o_{p}(1)$. Finally, in the notation of Lemma 2,

$$
T_{3, n}=\frac{n^{3 \frac{1}{2}}}{72} S_{n+1}^{-5} \sum_{i=2}^{\frac{n+1}{2}} f^{(4)}\left(\frac{2 i-2}{n+1}\right) Y_{i}=O_{p}\left(n^{-1 \frac{1}{2}}\right) \sum_{i=2}^{\frac{n+1}{2}} f^{(4)}\left(\frac{2 i-2}{n+1}\right) Y_{i} .
$$

From Lemma 2, we have

$$
\begin{gathered}
\mathbb{E} \sum_{i=2}^{\frac{n+1}{2}} f^{(4)}\left(\frac{2 i-2}{n+1}\right) Y_{i}=0, \\
\operatorname{Var} \sum_{i=2}^{\frac{n+1}{2}} f^{(4)}\left(\frac{2 i-2}{n+1}\right) Y_{i}=\sum_{i=2}^{\frac{n+1}{2}}\left(f^{(4)}\left(\frac{2 i-2}{n+1}\right)\right)^{2} \operatorname{Var} Y_{i}=O\left(n^{2}\right) .
\end{gathered}
$$


Now, Chebysev's inequality yields $T_{3, n}=o_{p}(1)$ and hence $T_{1, n}=o_{p}(1)$.

Proof of Theorem 2: Given the lemmas, especially Lemma 3, the proof of Theorem 2 is rather easy. If $\int_{0}^{1}\left(f^{(3)}(x)\right)^{2} d x=0$, then $f^{(3)}(x)=0$ for all $x \in[0,1]$ and hence trivially $I_{n}=I$, because $f$ is a polynomial of second degree. Therefore, we assume now $\int_{0}^{1}\left(f^{(3)}(x)\right)^{2} d x>0$. Using Lemma 1 we have

$$
\begin{aligned}
& \frac{n^{3 \frac{1}{2}}}{72} \sum_{i=1}^{\frac{n+1}{2}} f^{(3)}\left(\frac{2 i-2}{n+1}\right)\left(D_{2 i-1}+D_{2 i}\right)^{3}\left(D_{2 i}-D_{2 i-1}\right) \\
& \stackrel{d}{=} \frac{1}{72 \sqrt{2}}\left(\frac{n}{S_{n+1}}\right)^{4} \frac{1}{(n / 2)^{1 / 2}} \sum_{i=1}^{\frac{n+1}{2}} f^{(3)}\left(\frac{2 i-2}{n+1}\right)\left(E_{2 i-1}+E_{2 i}\right)^{3}\left(E_{2 i}-E_{2 i-1}\right) \\
& =:\left(\frac{n}{S_{n+1}}\right)^{4} W_{n} .
\end{aligned}
$$

By the weak law of large numbers and Lemma 3, it now remains to show Theorem 2 with $n^{3 \frac{1}{2}}\left(I_{n}-I\right)$ replaced by $W_{n}$. By Lemma 2 , we see that $\mathbb{E} W_{n}=0$ and

$$
\operatorname{Var} W_{n}=\frac{1}{2(72)^{2}} \frac{2}{n} \sum_{i=1}^{\frac{n+1}{2}}\left(f^{(3)}\left(\frac{2 i-2}{n+1}\right)\right)^{2} 120960 \rightarrow \frac{35}{3} \int_{0}^{1}\left(f^{(3)}(x)\right)^{2} d x
$$

Now, the Lindeberg central limit theorem applies, because of the boundedness of $\left|f^{(3)}\right|$, and it yields the result.

\section{Acknowledgements}

The authors are indebted to A.O.H. Axelsson and B.J.W. Polman for helpful discussions and their useful comments.

\section{References}

[1] Davis, P.J. and Rabinowitz, P., Methods of Numerical Integration, Academic Press, New York 1975.

[2] Isaacson, I. and Keller, H.B., Analysis of Numerical Methods, Wiley, New York 1966.

[3] Shorack, G.R. and Wellner, J.A., Empirical Processes with Applications to Statistics, Wiley, New York 1986. 


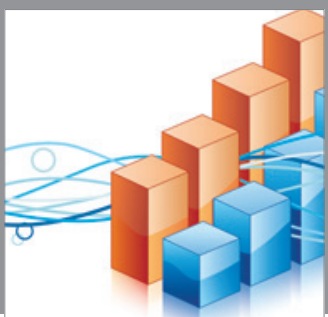

Advances in

Operations Research

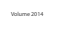

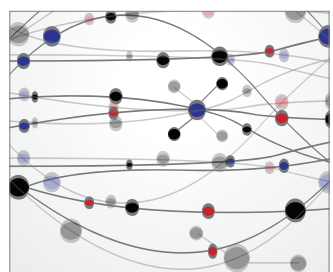

\section{The Scientific} World Journal
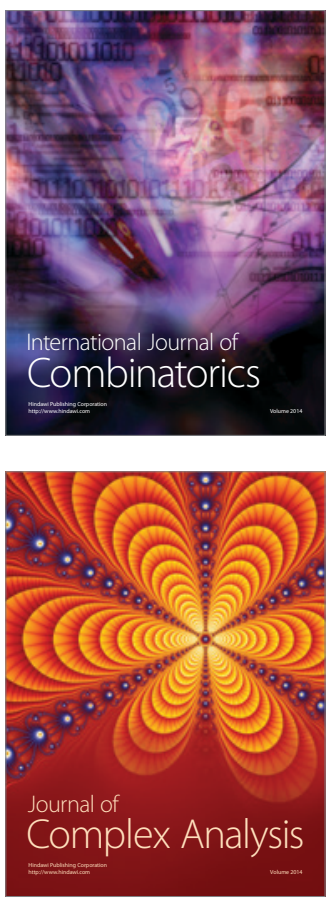

International Journal of

Mathematics and

Mathematical

Sciences
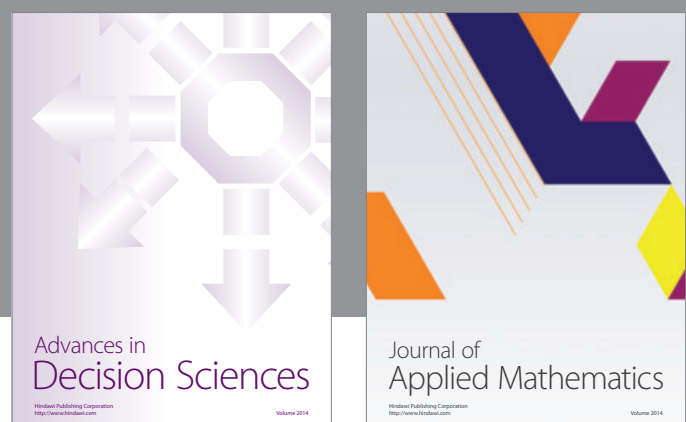

Journal of

Applied Mathematics
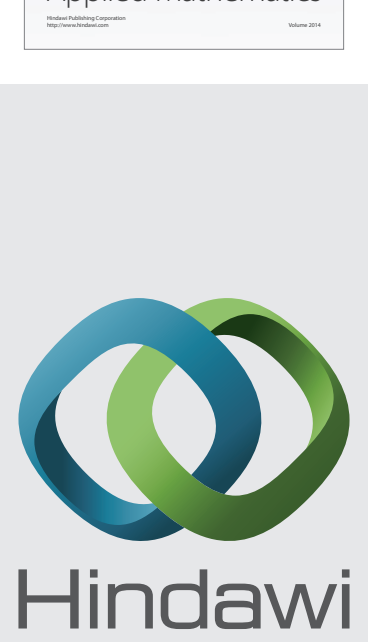

Submit your manuscripts at http://www.hindawi.com
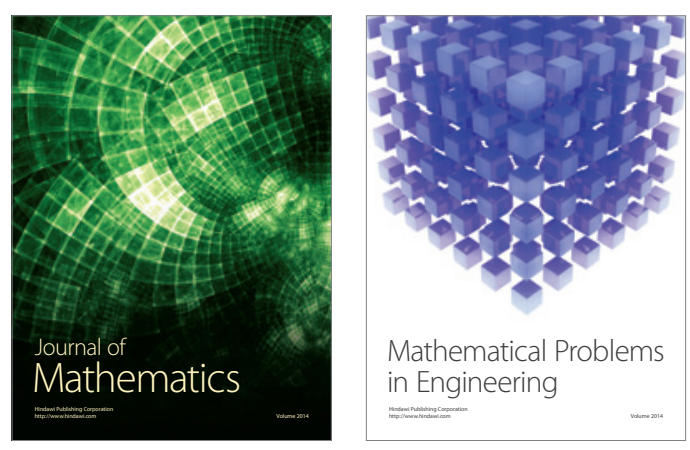

Mathematical Problems in Engineering
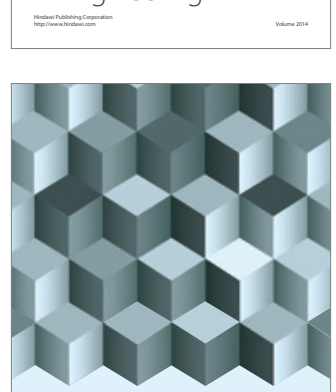

Journal of

Function Spaces
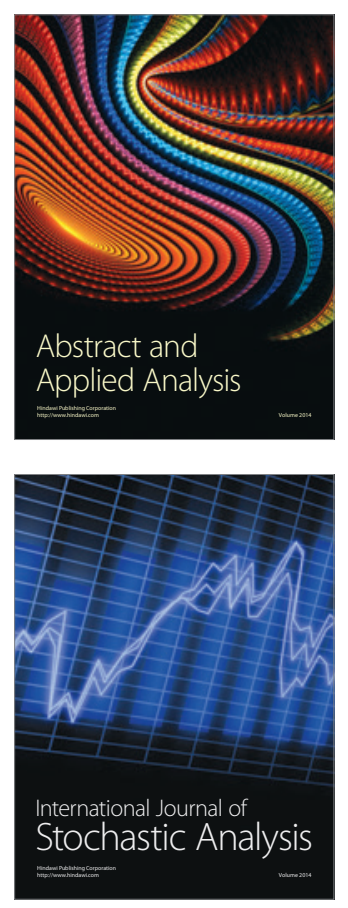

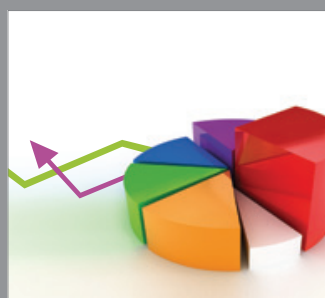

ournal of

Probability and Statistics

Promensencen
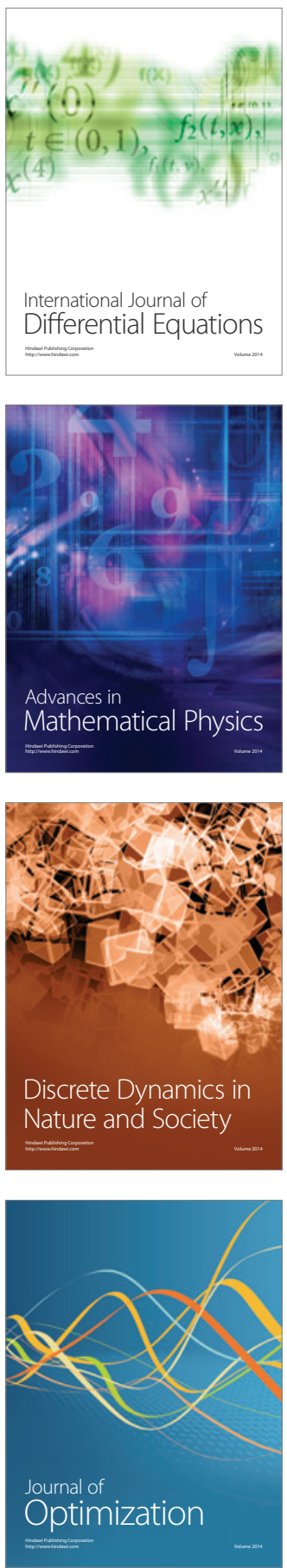